\title{
Is Schrödinger's Cat Alive?
}

\author{
Mani L. Bhaumik
}

Department of Physics and Astronomy, University of California, Los Angeles, USA. E-mail: bhaumik@physics.ucla.edu

Editors: Zvi Bern \& Danko Georgiev

Article history: Submitted on December 2, 2017; Accepted on December 9, 2017; Published on December 11, 2017.

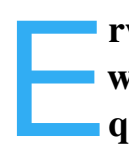
rwin Schrödinger is famous for presenting his wave equation of motion that jump-started quantum mechanics. His disenchantment with the Copenhagen interpretation of quantum mechanics led him to unveil the Schrödinger's cat paradox, which did not get much attention for nearly half a century. In the meantime, disappointment with quantum mechanics turned his interest to biology facilitating, albeit in a peripheral way, the revelation of the structure of DNA. Interest in Schrödinger's cat has recently come roaring back making its appearance conspicuously in numerous scientific articles. From the arguments presented here, it would appear that the legendary Schrödinger's cat is here to stay, symbolizing a profound truth that quantum reality exists at all scales; but we do not observe it in our daily macroscopic world as it is masked for all practical purposes, most likely by environmental decoherence with irreversible thermal effects.

Quanta 2017; 6: 70-80.

\section{Introduction}

Like most cats, this one slipped into the room practically unnoticed. Schrödinger's cat [1-5], which is supposedly both alive and dead at the same time, has by now attained a celebrated status in both scientific and public spheres. In addition to its surprisingly frequent mention in quantum physics articles, the quantum computation community

(c) $(9)$ This is an open access article distributed under the terms of the Creative Commons Attribution License CC-BY-3.0, which permits unrestricted use, distribution, and reproduction in any medium, provided the original author and source are credited. has bestowed it a permanent berth by coining a cat state in its repute. The appearance of a cat's image adorning the jacket of a popular undergraduate textbook [6] as well as two popular science books on the subject [7,8] speaks volumes about its privileged place in scientists' fancy. It has come to be the common metaphor for superposition so ubiquitous in the quantum domain. Perhaps because of its somewhat melodramatic character, it has also secured a place in popular culture with appearances in literature, television, film, music, cartoons, jokes, and video games. It would be rather fascinating to follow the trail of this captivating story since in a real sense Schrödinger's cat is a conspicuous symbolic entity representing a profound reality, namely: the manifest coexistence of the quantum and classical reality, which appear so particularly different in their attributes.

At the very dawn of the twentieth century, Max Planck initiated the quantum revolution by introducing the idea of a quantum [9]. After years of frustrating failure to work out an accurate formula for thermal radiation of a black body, Planck postulated that radiation energy is emitted and absorbed only in discrete packages he called quanta. But he was, however, a reluctant revolutionary and did not believe in the physical existence of the quantum, which so uniquely contributed in formulating the correct equation for the black body radiation.

It was Albert Einstein who recognized the reality of a quantum and suggested that radiation in space also consists of discrete quanta [10]. With near unanimity, the entire physics community_including Planck himselfremained very skeptical about the postulate since by then Maxwell's theory of electromagnetic radiation in terms of continuous wave motion had, through its numerous confirmations, become ingrained in people's minds. It 
also evoked the counter intuitive idea of a wave-particle duality. Nevertheless, Einstein unwaveringly persisted almost for two decades as the sole champion keeping the nascent quantum revolution alive. His tenacity was finally vindicated spectacularly by the discovery of the Compton effect in 1923, which demonstrated that X-rays could be deflected like billiard balls by an electron [11].

With an overwhelming support from Einstein, the young French graduate student, Louis de Broglie galvanized the quantum revolution in 1924 by boldly postulating in his doctoral thesis that even matter particles could have wave like properties. His hypothesis gained support from the fact that it could explain the radius of the stationary orbits of the electron in Niels Bohr's audacious model of the hydrogen atom, offered in 1913 [12-14]. The radius of a Bohr's conjectured orbit was an integral multiple of de Broglie's proposed wavelength of the electron. Years later, Einstein confided that he also came up with the idea of matter waves but did not publish it due to a lack of any evidence. After de Broglie submitted his thesis, Einstein ardently appealed to the physicists to look for an experimental proof of the postulated waves. Soon Clinton Davisson and Lester Germer provided the evidence in 1927 by observing a diffraction pattern in the beam of electrons scattered by nickel crystals [15].

In the meantime, through his correspondence with Einstein [16, p. 412], Erwin Schrödinger became fascinated by the radical concept of de Broglie's matter wave. At the request of Peter Debye, Schrödinger gave a seminar at ETH in Zurich toward the end of November 1925, enthusiastically explaining how the matter waves gave support to Niels Bohr's atomic model with stable electronic orbits. In the seminar, Debye expressed some skepticism about how waves, which normally spreads out, could confine itself in stable atomic orbits. He suggested that a relevant wave equation of motion should be formulated to deal with a wave in a proper way [16, pp. 419-421].

Schrödinger took up the challenge, since apparently he was already thinking about the subject himself, and promptly formulated his groundbreaking equation of motion for matter waves in early 1926 [17, 18]. With some assistance from his associate, mathematical physicist Hermann Weyl, Schrödinger found the solutions for the standing waves of the discrete orbits of the hydrogen atom [19. p. 1165]. Schrödinger stated in his first paper

The essential thing seems to me to be, that the postulation of "whole numbers" no longer enters into the quantum rules mysteriously, but that we have traced the matter a step further back, and found the "integralness" to have its origin in the finiteness and single-valuedness of a certain space function. [18, p. 9]
The astounding success of his equation in giving precise explanation of the quantized orbital motion of an electron in a hydrogen atom initiated a storm of activities and the quantum revolution went into full swing.

\section{Trail to the Schrödinger Equation}

As we have just described, after the demonstration of the Compton effect, wave-particle duality of massless particles like photons was established beyond a reasonable doubt. Louis de Broglie's profound insight to extend the idea to massive particles was a feat of genius. He correctly surmised that a massive particle like an electron would have a characteristic internal frequency $v_{0}$ in compliance with the Planck-Einstein relation

$$
E=m_{0} c^{2}=h v_{0}
$$

that would obey the rules of special relativity to provide the length of the matter wave $\lambda=h / p$ or equivalently $p=\hbar k$ analogous to that of the photon. However, he struggled with a derivation of the matter wave using the special theory of relativity to such an extent that he portrayed the matter wave to be merely a fictitious guiding wave to be used for the kinematics of the matter particle. Consequently, the matter wave proposition received the moniker de Broglie's hypothesis instead of a theory. We now know that the relationship $p=\hbar k$ can be elegantly comprehended using the four-vector procedure of the special theory of relativity.

In an earlier presentation [20], it was shown that the wave packet of a quantum particle could be deduced from an objective reality to be given by

$$
\psi(x, t)=\frac{1}{\sqrt{2 \pi}} \int_{-\infty}^{\infty} \tilde{\psi}(k) e^{l\left(k x-\omega_{k} t\right)} d k
$$

The group velocity of the wave-packet for a massless particle like a photon is

$$
v_{g}=\frac{d \omega}{d k}=\frac{d(k c)}{d k}=c
$$

For a massive particle like an electron, the group velocity of the wave packet in Eq. (2) is no longer equal to the constant $c$, but a variable velocity $v$ depending upon its energy-momentum. Accordingly, the spacetime dependence $e^{l\left(k x-\omega_{k} t\right)}$ must change frequently, which can be deduced from the special theory of relativity.

The transformation of the momentum four-vector $\left(\frac{E}{c}, \vec{p}\right)$ and the wave four-vector $\left(\frac{\omega}{c}, \vec{k}\right)$ that keeps their magnitude invariant is the Lorentz transformation. Considering a laboratory frame $S$ and a rest frame $S^{\prime}$ with a 
boost velocity $v$ in the $x$ direction, the Lorentz transformation relations for the momentum four-vector and the wave four-vector are

$$
\begin{aligned}
\frac{E^{\prime}}{c} & =\gamma\left(\frac{E}{c}-\beta p_{x}\right) \\
p_{x}^{\prime} & =\gamma\left(p_{x}-\beta \frac{E}{c}\right) \\
p_{y}^{\prime} & =p_{y} \\
p_{z}^{\prime} & =p_{z}
\end{aligned}
$$

and

$$
\begin{aligned}
\frac{\omega^{\prime}}{c} & =\gamma\left(\frac{\omega}{c}-\beta k_{x}\right) \\
k_{x}^{\prime} & =\gamma\left(k_{x}-\beta \frac{\omega}{c}\right) \\
k_{y}^{\prime} & =k_{y} \\
k_{z}^{\prime} & =k_{z}
\end{aligned}
$$

where $\beta=\frac{v}{c}$ and $\gamma=1 / \sqrt{1-\beta^{2}}$.

To determine the proportionality constant between the two four-vectors, we correlate their timelike components after multiplying the component of the wave four-vector by $\hbar$ :

$$
\begin{aligned}
\frac{E^{\prime}}{c} & =\gamma\left(\frac{E}{c}-\beta p_{x}\right) \\
\frac{\hbar \omega^{\prime}}{c} & =\gamma\left(\frac{\hbar \omega}{c}-\beta \hbar k_{x}\right)
\end{aligned}
$$

Subtracting Eq. (13) from Eq. (12), we find

$$
\frac{E^{\prime}}{c}-\frac{\hbar \omega^{\prime}}{c}=\gamma\left(\frac{E}{c}-\frac{\hbar \omega}{c}-\beta p_{x}+\beta \hbar k_{x}\right)
$$

Since the laws of physics are the same in all inertial frames of reference, the Planck's law $E=\hbar \omega$ in frame $S$ should hold true in frame $S^{\prime}$, giving us $E^{\prime}=\hbar \omega^{\prime}$. Thus Eq. (14) reduces to

$$
\gamma \beta\left(p_{x}-\hbar k_{x}\right)=0
$$

According to the zero product property of algebra, either $\gamma \beta=0$ or $p_{x}-\hbar k_{x}=0$. Because $\gamma \beta$ is non-zero, we obtain

$$
p_{x}=\hbar k_{x}
$$

Or more generally, $p=\hbar k$ irrespective of the mass of the particle, zero or otherwise. This relationship for a massive particle is the celebrated de Broglie hypothesis. However, we now realize that the relationship can indeed be derived from fundamental considerations and does not need to be a mere hypothesis.

As presented earlier [21], using the relationship in Eq. (9) the group velocity of the wave packet is $v_{g}=v$, which is the velocity of the "particle" represented by the wave packet. Therefore, the wave packet moves with the velocity of either a massive or a massless particle. Steven Weinberg also confirms this result using a slightly different consideration [22, pp. 11-12].

\section{Kinematics of the wave packet}

Following Schrödinger, the equation of motion for the wave packet given in Eq. (2) can now be formulated using the relationship in Eq. (16).

Schrödinger's astute intuition was to express the phase of a plane wave utilizing Eq. (16) to achieve

$$
\Psi(x, t)=A e^{l(k x-\omega t)}=A e^{\frac{l}{\hbar}(p x-E t)}
$$

and to realize that the first order partial derivatives were: with respect to space

$$
\begin{gathered}
\frac{\partial \Psi}{\partial x}=\frac{l}{\hbar} p A e^{\frac{l}{\hbar}(p x-E t)}=\frac{l}{\hbar} p \Psi \\
p \Psi=-\imath \hbar \frac{\partial \Psi}{\partial x}
\end{gathered}
$$

thus the momentum operator is

$$
\hat{p}=-\imath \hbar \frac{\partial}{\partial x}
$$

and with respect to time

$$
\frac{\partial \Psi}{\partial t}=-\frac{l}{\hbar} E A e^{\frac{l}{\hbar}(p x-E t)}=-\frac{l}{\hbar} E \Psi
$$

or

$$
E \Psi=\imath \hbar \frac{\partial \Psi}{\partial t}
$$

thus the energy operator is

$$
\hat{E}=\imath \hbar \frac{\partial}{\partial t}
$$

for any state of energy $E$.

Max Born generalized this relationship for any system described by a Hamiltonian $\hat{H}$. The time dependence of any wave function, whether or not for a state of definite energy, is then given by

$$
\imath \hbar \frac{\partial \Psi}{\partial t}=\hat{H} \Psi
$$

The equation 24 is known as the general Schrödinger equation. It is indeed quite general and used throughout quantum mechanics, for everything from the Dirac equation to quantum field theory, by utilizing various complicated expressions for the Hamiltonian $\hat{H}$.

If the Hamiltonian itself is not explicitly dependent on time, Eq. (22) shows that

$$
\hat{H} \Psi=E \Psi
$$

For a single non-relativistic particle of mass $m$, its energy in a potential $V(x)$ is $\frac{p^{2}}{2 m}+V(x)$ giving us

$$
-\frac{\hbar^{2}}{2 m} \nabla^{2} \Psi(x)+V(x) \Psi(x)=E \Psi(x)
$$


This is the time-independent Schrödinger equation for a single non-relativistic particle of energy $E$. The equation (26) solved for the hydrogen atom, with the three boundary conditions that: $\Psi(x)$ is single-valued, $\Psi(x)$ returns to the same value if $x$ goes around a closed curve, and $\Psi(x)$ vanishes as the magnitude of $x$ goes to infinity, accurately reproduced Bohr's formula for the discrete energy levels but without any assumption.

Einstein wholeheartedly endorsed the paper since he considered the wave nature of particle gave a clearer intuitive picture as compared to the rather abstract matrix mechanics introduced by Werner Heisenberg, Max Born and Pasqual Jordan in 1925. In rapid succession within a few months after his first paper, Schrödinger was able to demonstrate that the analytical solution of his time independent wave equation indeed predicts the discrete energy levels of several other non-relativistic quantum systems, among them, the quantum harmonic oscillator, diatomic molecule, and Stark effect [18, 23]. With Einstein's commendation and the immense success of the wave equation, Schrödinger equation became the benchmark for wave mechanics at the time bestowing its inventor an instant renown. The very following year, he was honored with the venerable chair formerly held by Max Planck at the Friedrich Wilhelm University in Berlin.

After the spectacular success of the time independent wave equation, Schrödinger focused his attention on developing a time dependent equation for treating problems in which the quantum system changes with time as in scattering problems. Using the total energy $H=\frac{p^{2}}{2 m}+V(x)$ for a single non-relativistic particle in a potential $V(x)$, and taking the square of the momentum operator, Schrödinger soon unveiled his time dependent equation of motion for a wave packet

$$
\imath \hbar \frac{\partial \Psi(x, t)}{\partial t}=\hat{H} \Psi(x, t)=-\frac{\hbar^{2}}{2 m} \nabla^{2} \Psi(x, t)+V(x) \Psi(x, t)
$$

Clearly, the plane wave in Eq. (17) is a solution of the Schrödinger equation (27). However, the Schrödinger equation is a linear differential equation. So a linear combination of plane waves is also a valid solution. The wave packet in Eq. (2) is just such a linear combination. The momentum wave function $\tilde{\psi}(k)$ appearing in the integrand is an integral of the position wave functions since the position and momentum space wave functions are Fourier transforms of each other. Therefore the kinematics of the wave packet described in Eq. (2) can be processed using the Schrödinger equation (27).

This distinctive aspect of superposition of solutions of the linear Schrödinger equation is its unique signature. It should not be surprising since in our daily lives, we do see water wavelets superpose when they come in contact.
In the quantum domain as well, we see chargeless bosons like photons and gravitons exhibit superposition by producing macroscopic classical waves. But the fermion wave functions, being antisymmetric, in addition to some of them having different charges, are not alike and do not produce classical waves. However, the bizarre implication of superposition, especially as construed by the Copenhagen interpretation for measurement, made both Einstein and Schrödinger, the pioneering leaders of the quantum revolution, enormously unhappy.

\section{Probability Amplitude}

In his initial studies involving the time independent wave equation to predict energies of discrete quantum states, Schrödinger did not have to be too concerned about what exactly the wave function $\Psi(x, t)$ did represent. It would be tempting to think, as in fact Schrödinger originally did, that the wave function represented a smeared out charge distribution of the electron. In an experiment involving the scattering of electrons, Max Born realized that Schrödinger's contention that the wave function represented a charge distribution could not be sustained [24]. Instead he suggested as it appears in his Nobel lecture

Again an idea of Einstein's gave me the lead. He had tried to make the duality of particleslight quanta or photons - and waves comprehensible by interpreting the square of the optical wave amplitudes as probability density for the occurrence of photons. This concept could at once be carried over to the $\psi$-function: $|\psi|^{2}$ ought to represent the probability density for electrons (or other particles). [25]

This is known as the Born's rule.

But recalling how the wave packet had come to be [20], it would have been obvious that Born was correct. The wave packet consists of irregular disturbances, the sum total of which represents the mass, energy momentum, charge of a particle like electron. Therefore, the wave function is in fact a function of probability amplitude for finding the particle.

It should be noted, however, that although the attributes of the various irregular disturbances are mostly characteristics of their respective quantum fields with different charge, spin, etc., they have one aspect in common. The element of disturbance in energy is identical for all fields. Energy density of a wave is given by the square of its amplitude. Therefore, to get the probability density, we have to take the square of the amplitude of the wave function, which usually involves a complex quantity. Consequently, the square of the amplitude $\Psi^{*}(x, t) \Psi(x, t)$, which is the 
probability density function $P(x, t)$, should represent the probability density for finding a particle in position space at time $t$. As discussed earlier, this is acknowledged as the renowned Born's rule, which is a necessary hypothesis of quantum mechanics. But as we have just presented, it is a natural consequence of the reality of the wave function revealed in a previous article [20] and does not need to be a hypothetical rule.

However, it is of critical importance that the wave function is normalized

$$
\int_{-\infty}^{\infty} \Psi^{*}(x, t) \Psi(x, t) d x=1
$$

since the equation describes one particle with the sum of probability to be 1 . Born's probabilistic description of the wave function was promptly taken over by Niels Bohr in Copenhagen who in essence became the father of the long-reigning Copenhagen interpretation of quantum mechanics.

According to the Copenhagen interpretation, physical systems in superposed states do not possess real properties prior to being measured, and quantum mechanics can only predict the probabilities that measurements will produce certain results. The act of measurement using a macroscopic device by an observer collapses the wave function, causing the set of probabilities to reduce to only one of the possible values.

It is well known that both pioneers of quantum mechanics, Einstein and Schrödinger, were extremely troubled by the Copenhagen interpretation. Much has been written on the famous Bohr-Einstein debates. The concerns of Einstein have been discussed in greater detail in an earlier communication [20]. His most strident objection was the almost cult-like denial by the proponents of the Copenhagen interpretation of any reality in a quantum system prior to measurement. Among others, in one noteworthy sarcastic comment he states

The present quantum theory is unable to provide the description of a real state of physical facts, but only of an (incomplete) knowledge of such. Moreover, the very concept of a real factual state is debarred by the 'orthodox' quantum theoreticians. The situation arrived at corresponds almost exactly to that of the good old Bishop Berkeley. [26, pp. 73-74]

He expected the proponents to be at least open, as he himself was, to the possibility that quantum mechanics as currently formulated might be incomplete.

Schrödinger's main objection appears to be the rather fictional depiction of the superposed states and their probability interpretation as well as the collapse of the wave function. He envisioned that after a unitary evolution of his wave equation, it could be somehow extrapolated up to the macroscopic scale of the measuring device. Sometime later, he sarcastically commented that

I don't like it, and I'm sorry I ever had anything to do with it. [7, p. v]

\section{Quantum Entanglement}

Einstein expressed his dismay regarding the implications of quantum mechanics very publicly through the famous Bohr-Einstein debates beginning at the Solvay conference in 1927, of which Bohr was the generally supposed winner. Bohr, meanwhile, was troubled by none of the elements that appalled Einstein. He made his own peace with the contradictions by asserting a principle of complementarity that emphasized the role of the observer over the observed and placed limits on what the observer could know about a quantum system. Given his disenchantment with the Copenhagen interpretation, Einstein's attention shifted from quantum mechanics primarily toward his interest in developing a unified theory of gravity and electro-magnetism. After the well-known turbulent times in Germany, Einstein left Europe and in 1933, permanently settled down at the Institute for Advanced Study in Princeton, where he finally had the time and serenity to resume his thoughts on quantum mechanics.

In 1935, Albert Einstein together with Boris Podolsky and Nathan Rosen published the seminal EPR paper in an attempt to show that the description of reality as given by a wave function was not complete [27]. Einstein tried to illustrate that superposition of the quantum states of two particles cannot be real. Because when the particles are separated by an arbitrarily large distance, measuring a particular property of one of the particles would instantly affect the other. In a letter to Born, Einstein wrote

I admit, of course, that there is a considerable amount of validity in the statistical approach which you were the first to recognize clearly as necessary given the framework of the existing formalism. I cannot seriously believe in it because the theory cannot be reconciled with the idea that physics should represent a reality in time and space, free from spooky actions at a distance. [28, p. 158]

Because of Einstein's repute, the EPR article generated some impressive newspaper headlines. But the physics community essentially ignored it for quite a while. Later, in the 1950s, David Bohm tried to show that Einstein's desired element of reality could be supported by existence of some local hidden variables. Bohm's ideas met with stiff resistance from the scientists. 
It was not until 1964, when John Bell started his seminal analysis known as Bell's inequality that helped to discard the existence of local hidden variables, proposed by Bohm, but indicated the existence of nonlocality revealed in Bohm's investigation. Bell ingenuously proposed an approach to experimentally verify nonlocality between a pair of quantum particles in superposed states, which are now called entangled states as coined by Schrödinger. Numerous experimental verifications of the existence of quantum entanglement beyond any reasonable doubt have now been accomplished establishing that quantum superposition is undoubtedly real. Using a satellite, Chinese scientists have demonstrated entanglement over a distance as large as 1200 kilometers patently attesting that Einstein's "spooky action at a distance" is here to stay [29]. He still might have derived some satisfaction from knowing that in conformity with his special theory of relativity, no meaningful signal can be sent using entanglement and the spookiness of the action is circumscribed.

As explained by Narnhofer and Thirring, in quantum field theory almost everything is entangled in the quantum and the mesoscopic domains [30]. Penrose finds this extremely puzzling, stating

\begin{abstract}
Since, according to quantum mechanics, entanglement is such a ubiquitous phenomenonand we recall that the stupendous majority of quantum states are actually entangled oneswhy is it something that we barely notice in our direct experience in the world? [31, p. 591]
\end{abstract}

It is rather ironic that the very pivotal EPR thought experiment in which Einstein attempted in effect to show the impossibility of quantum entanglement would lead to one of the most astounding discoveries of the twentieth century. It has opened a floodgate of activities in quantum physics. The possible uses of quantum entanglement in a variety of novel applications such as quantum cryptography, quantum computation, and quantum teleportation have become areas of very active research.

It has also provided an immense stimulus to basic scientific investigations. Experts such as Maldacena and Susskind postulate that ER=EPR implying there is an as yet unknown quantum mechanical version of a classical wormhole that permits quantum entanglement [32]. There is a possibility that the quantum fluctuations of the fields are themselves entangled facilitating a quantum mechanical Einstein-Rosen bridge [33]. Most exciting perhaps is the intriguing prospect that spacetime itself could be stitched together by entanglement [34, 35].

\section{Arrival of the Schrödinger's Cat}

Like Einstein, Schrödinger fled the rapidly deteriorating political situation and left Berlin in 1933 for England to join the University of Oxford. Soon after he arrived, he received the Nobel Prize, which should have augured a very comfortable existence for him. Instead, the glare that focused on his personal life and unconventional marital arrangement outshone the Nobel Prize and caused him considerable difficulty in securing a tenured position until 1939, when he became the Director of the Institute for Advanced Studies in Dublin. In the midst of the turmoil of his tenure issues in 1935, during extensive correspondence with Albert Einstein, he proposed what is now called the Schrödinger's cat thought experiment.

As one of the doctrines of the Copenhagen interpretation, a system stops being in a superposition of states and becomes one or the other, only when an observation takes place, which collapses the entire wave function. Both Einstein and Schrödinger thought this to be downright preposterous.

In the same period of time during which Einstein presented his EPR paper, he also wrote to Schrödinger comparing the absurdity of the Copenhagen interpretation to the notion of a keg of gun powder that is simultaneously both exploded and unexploded. In an attempt to take Einstein's thoughts a step further, the famed creator of wave mechanics conjured up the enigmatic Schrödinger's cat, which by virtue of being unobservable inside a box and subject to a random quantum trigger that may or may not kill the kitty, is put into a quantum superposition of being alive and dead at the same time.

Einstein was duly impressed and wrote in a letter to Schrödinger a bit later:

You are the only contemporary physicist, besides Laue, who sees that one cannot get around the assumption of reality, if only one is honest. Most of them simply do not see what sort of risky game they are playing with realityreality as something independent of what is experimentally established. They somehow believe that the quantum theory provides a description of reality, and even a complete description; this interpretation is, however, refuted most elegantly by your system of radioactive atom + Geiger counter + amplifier + charge of gun powder + cat in a box, in which the $\psi$-function of the system contains both the cat alive and blown to bits. Nobody really doubts that the presence or absence of the cat is something independent of the act of observation.

[36, p. 39] 
Although Schrödinger loved animals and apparently had a cat named Milton at the time he devised his paradox, no one knows why he chose a cat and not a dog or something else. Schrödinger's thought experiment, however, had little impact during his lifetime. From the time he proposed it and till his death, it was scarcely mentioned in the literature. Even Schrödinger rarely brought it up. After its rather clandestine appearance, Schrödinger's cat went into a long slumber of nearly half a century.

The absurd scenario portrayed in the thought experiment remained mostly an academic curiosity until the 1980s, when it was realized that, under suitable conditions, a macroscopic object with many microscopic degrees of freedom could behave quantum mechanically, provided that it was sufficiently decoupled from its environment. Such decoupling is required because quantum superposition rapidly decoheres as a result of complicated interactions with the environment and its inherently irreversible thermal processes.

In the 1980s, there were also some other very exciting developments. The French physicist Alain Aspect, in 1982, gave a quite definitive experimental demonstration of quantum entanglement that led to a burst of activities [37,38]. In 1984, Charles Bennet and Gilles Brassard proposed a theoretical system for quantum cryptography using photons in a superposition state to create a secure key distribution [39]. Achievement of a mesoscopic Schrödinger's cat state allowing potential studies of quantum decoherence generated considerable enthusiasm as well.

The explosion of publication of research articles on these subjects is too voluminous to describe here. An extensive list of publications on decoherence can be found in papers by its prominent investigator, W. H. Zurek [40-43], as well as in the book by M. Schlosshauer and his other papers [44-46]. Only a selection of especially pertinent papers on Schrödinger's cat will now be discussed.

The physics Nobel Laureates Serge Haroche and David Wineland have allowed Schrödinger's cat to roam the most prestigious halls by mentioning it in their Nobel lectures [47,48]. Wineland shared the Nobel Prize with Haroche in 2012 "for ground breaking experimental methods that enable measuring and manipulation of individual quantum systems." They independently developed methods for measuring and manipulating individual particles while preserving their quantum mechanical nature in ways that were previously thought unachievable. The two researchers have taken different routes to the study of some of the same phenomena.

Wineland and his group used a trapped beryllium ion, laser cooled to its zero-point energy [49]. A Schrödinger's cat state was ingenuously produced by applying a sequence of laser pulses to create superposition of the ion's internal hyperfine electronic states with its external positional states resulting from its zero-point motion in the potential well of the trapping electro-magnetic field. A mesoscopic distance of more than $80 \mathrm{~nm}$ separating the individual wave packets of about $7 \mathrm{~nm}$ was accomplished creating a mesoscopic Schrödinger's cat state that could allow controlled studies of quantum decoherence and study of the quantum-classical boundary. Quantum decoherence has received great interest to find a realistic solution of the long standing measurement problem and more recently for application to quantum computing [50,51] and quantum cryptography [52].

Of necessity, to measure the outcome of any quantum system we have to use a macroscopic device composed of many quantum particles interacting in an incredibly complicated way, and also partaking in irreversible thermal processes. Is it then surprising that the transition from the quantum to classical domain is the most difficult as well as a controversial subject in quantum physics?

Fortunately, with the help of many sophisticated contemporary experiments accompanied by highly developed theoretical investigations, significant progress is being achieved although a very substantial difference exists between theoretically anticipated and experimentally observed values of decoherence time. A consensus seems to be developing in favor of the environmental decoherence model pioneered by Zurek [40-43]. According to his theoretical analysis, the decoherence time of a macroscopic object with a mass of $1 \mathrm{~g}$ at a temperature of $300 \mathrm{~K} \mathrm{ex}-$ tended over a distance of $1 \mathrm{~cm}$ could be as short as $10^{-23} \mathrm{~s}$. This, however, appears to be far too short compared to some recent experimental investigations.

Observation has confirmed that quantum coherence is sustained for longer than a few picoseconds inside photosynthetic light harvesting pigment-protein complexes at room temperature [53-60]. Even more spectacular are analyses of recent experimental studies of the avian compass [61]. Quantum superposition and entanglement are sustained in this living system for at least tens of microseconds. Similar time frames for sustaining quantum superposition have been conjectured in the tubulin molecules of microtubules in the cytoskeletons of living organisms [62].

These observations are strikingly at variance with the view that life is too warm and wet for such quantum phenomena to endure over such a relatively long time. It would appear that living entities may develop at least in particular locations some sorts of shielding mechanism to protect quantum superposition from environmental decoherence. But this leaves us in a quandary about how long quantum superposition could last in a living cat weighing at least $5 \mathrm{~kg}$ and containing some $10^{27}$ atoms. In the absence of any meaningful data, the best educated 
guess would be that decoherence will perhaps occur in extremely short time scale under ambient conditions.

The coherence of superposition in large quantum objects is usually demonstrated by a double slit type diffraction experiment. Such studies have been performed with molecules as large as $1 \mathrm{~nm}$, namely carbon 60 Bucky balls [63]. Recently, a diffraction pattern has been observed in experiment of superposed molecules containing 10000 atomic mass units [64].

Most remarkably, using a rather sophisticated procedure, coherent superposition has been demonstrated in a macroscopic object containing an estimated 10 trillion atoms [65]. For this purpose, the investigators used a 40 micrometer long mechanical resonator, just large enough to be visible with the naked eye. The resonator with a resonant frequency of $6.175 \mathrm{GHz}$ to its first excited phonon state was cooled to a temperature of merely $25 \mathrm{mK}$ over absolute zero and put in a very high vacuum to minimize environmental decoherence. Under these circumstances, the resonator was confirmed to be in its ground state. Then a signal from a coupled qubit possessing the resonance frequency of $6.175 \mathrm{GHz}$ was injected into the resonator thereby transferring the superposition feature of the qubit to the macroscopic object. Superposition of the ground and the first excited phonon state of the macroscopic resonator lasted for the resonator relaxation time of $6.1 \mathrm{~ns}$.

The above demonstration provides strong evidence that quantum mechanics and its attendant aspect of superposition applies to macroscopic objects and can be revealed under appropriate circumstances. Can it apply to Schrödinger's cat? The answer in principle should be yes-at least at an extremely short time scale. But to prove it, the cat will surely perish for other reasons! Because in order to conduct the experiment, it would be necessary to remove all sources of environmental decoherence by exposing the cat to exceptionally low temperatures and high vacuum and perhaps stopping irreversible metabolic processes as well.

\section{Conclusion}

By now the Schrödinger's cat has been in existence for over eight decades surviving longer than the mythical cat's nine lives. Considering the fact that quantum superposition has been convincingly established in quantum, mesoscopic and a macroscopic domain, Schrödinger's cat is likely to live on, symbolizing a profound truth that quantum reality exists in all scales. We do not observe it in our daily macroscopic world because it is masked for all practical purposes, predominantly by environmental decoherence possessing irreversible thermal effects.
It should be noted however, that the concerns, which brought the Schrödinger's cat into existence are on their way out. We have cogently pointed out that the probabilistic feature of a quantum particle is an inherent aspect arising out of the genesis of the particle as a wave packet comprising a collection of irregular disturbances of the underlying quantum fields [20]. This is in addition to the innate uncertainty relation $\Delta x \Delta p \geq \frac{1}{2} \hbar$ of a real wave packet. Comprehension of the reality of the wave packet acting as a particle was possible only after the development of the standard model in quantum field theory and was thus unavailable to both Einstein and Schrödinger and to the framers of the Copenhagen interpretation.

Bohr's contention that there is no evocative reality before measurement collapses the wave function was not entirely without merit. But his emphatic denial of any reality whatsoever appears unjustifiable. His emphasis on the critical role of a conscious observer for measurement is also untenable, since the universe developed to a fairly mature state obeying quantum rules long before the possibility of emergence of conscious beings. Einstein's insistence on the existence of reality in all scales from quantum to classical would be correct, if only we allow him the understandable failure to see that the quantum reality is not necessarily the same as the classical reality. Had the Bohr-Einstein debate take place today, it probably would have been declared a draw!

\section{Acknowledgement}

The author wishes to thank Joseph Rudnick and Zvi Bern for helpful discussions.

\section{References}

[1] Schrödinger E. Die gegenwärtige Situation in der Quantenmechanik. I. Naturwissenschaften 1935; 23(48): 807-812. doi : 10.1007/bf01491891

[2] Schrödinger E. Die gegenwärtige Situation in der Quantenmechanik. II. Naturwissenschaften 1935; 23(49): 823-828. doi : 10. 1007/bf01491914

[3] Schrödinger E. Die gegenwärtige Situation in der Quantenmechanik. III. Naturwissenschaften 1935; 23(50): 844-849. doi : 10.1007/bf01491987

[4] Trimmer JD. The present situation in quantum mechanics: a translation of Schrödinger's "cat paradox" paper. Proceedings of the American Philosophical Society 1980; 124(5): 323-338. JSTOR: 986572 
[5] Schrödinger E. The present situation in quantum mechanics: a translation of Schrödinger's "cat paradox" paper. In: Quantum Theory and Measurement. Wheeler JA, Zurek WH (editors), Trimmer JD (translator), New Jersey: Princeton University Press, 1983, pp. 152-167.

[6] Griffiths DJ. Introduction to Quantum Mechanics, 2nd edition. Upper Saddle River, New Jersey: Prentice Hall, 2004.

[7] Gribbin J. In Search of Schrödinger's Cat: Quantum Physics and Reality. New York: Bantam Books, 1984.

[8] Rowlands P. How Schrödinger's Cat Escaped the Box. Singapore: World Scientific, 2015. doi:10. $1142 / 9391$

[9] Planck M. Ueber das Gesetz der Energieverteilung im Normalspectrum. Annalen der Physik 1901; 309(3): 553-563. doi:10.1002/andp. 19013090310

[10] Einstein A. Über einen die Erzeugung und Verwandlung des Lichtes betreffenden heuristischen Gesichtspunkt. Annalen der Physik 1905; 17(6): 132-148. doi:10.1002/andp.19053220607

[11] Compton AH. A quantum theory of the scattering of X-rays by light elements. Physical Review 1923; 21(5): 483-502. doi: 10.1103/PhysRev.21.483

[12] Bohr N. On the constitution of atoms and molecules. Part I. Philosophical Magazine 1913; 26(151): 125. doi : $10.1080 / 14786441308634955$

[13] Bohr N. On the constitution of atoms and molecules. Part II. Systems containing only a single nucleus. Philosophical Magazine 1913; 26(153): 476-502. doi : $10.1080 / 14786441308634993$

[14] Bohr N. On the constitution of atoms and molecules. Part III. Systems containing several nuclei. Philosophical Magazine 1913; 26(155): 857-875. doi : 10.1080/14786441308635031

[15] Davisson C, Germer LH. Diffraction of electrons by a crystal of nickel. Physical Review 1927; 30(6): 705-740. doi:10.1103/PhysRev. 30.705

[16] Mehra J, Rechenberg H. The Historical Development of Quantum Theory. Vol. 5. Erwin Schrödinger and the Rise of Wave Mechanics. Part 2. The Creation of Wave Mechanics: Early Response and Applications 1925-1926. New York: Springer, 1987.

[17] Schrödinger E. Quantisierung als Eigenwertproblem: Erste Mitteilung. Annalen der Physik
1926; 4(79): 361-376. doi:10.1002/andp. 19263840404

[18] Schrödinger E. Collected Papers on Wave Mechanics. London: Blackie \& Son, 1928. https://archive.org/details/in.ernet. dli.2015.220691

[19] Mehra J. Erwin Schrödinger and the rise of wave mechanics. II. The creation of wave mechanics. Foundations of Physics 1987; 17(12): 1141-1188. doi:10.1007/bf01889592

[20] Bhaumik ML. Was Albert Einstein wrong on quantum physics? Quanta 2015; 4(1): 35-42. arXiv: 1511.05098, doi:10.12743/quanta.v4i1.47

[21] Bhaumik ML. Deciphering the enigma of waveparticle duality. Quanta 2016; 5(1): 93-100. arXiv: 1611.00226, doi:10.12743/quanta.v5i1.54

[22] Weinberg S. Lectures on Quantum Mechanics. Cambridge: Cambridge University Press, 2013.

[23] Schrödinger E. An undulatory theory of the mechanics of atoms and molecules. Physical Review 1926; 28(6): 1049-1070. doi : 10.1103/PhysRev. 28.1049

[24] Born M. Zur Quantenmechanik der Stoßvorgänge. Zeitschrift für Physik A 1926; 37(12): 863-867. doi : 10.1007/BF01397477

[25] Born M. The statistical interpretation of quantum mechanics. Nobel Lecture, December 11, 1954: http://www.nobelprize.org/ nobel_prizes/physics/laureates/1954/ born-lecture.pdf

[26] Jammer M. Einstein and quantum physics. In: Albert Einstein, Historical and Cultural Perspectives: The Centennial Symposium in Jerusalem. Holton G, Elkana Y (editors), Princeton: Princeton University Press, 1982, pp. 59-76. doi:10.2307/j. ctt7zvrpt.7

[27] Einstein A, Podolsky B, Rosen N. Can quantummechanical description of physical reality be considered complete? Physical Review 1935; 47(10): 777-780. doi : 10.1103/PhysRev.47.777

[28] Born M, Einstein A. The Born-Einstein Letters: Correspondence Between Albert Einstein and Max and Hedwig Born from 1916-1955, with Commentaries by Max Born. Born I (translator), London: Macmillan, 1971. http://archive.org/ details/TheBornEinsteinLetters 
[29] Yin J, Cao Y, Li Y-H, Liao S-K, Zhang L, Ren J-G, Cai W-Q, Liu W-Y, Li B, Dai H, Li G-B, Lu Q-M, Gong Y-H, Xu Y, Li S-L, Li F-Z, Yin Y-Y, Jiang Z-Q, Li M, Jia J-J, Ren G, He D, Zhou Y-L, Zhang X-X, Wang N, Chang X, Zhu Z-C, Liu N-L, Chen Y-A, Lu C-Y, Shu R, Peng C-Z, Wang J-Y, Pan J-W. Satellite-based entanglement distribution over 1200 kilometers. Science 2017; 356(6343): 1140-1144. doi:10.1126/science. aan3211

[30] Narnhofer H, Thirring W. Entanglement, Bell inequality and all that. Journal of Mathematical Physics 2012; 53(9): 095210. doi:10.1063/1. 4738376

[31] Penrose R. The Road to Reality: A Complete Guide to the Laws of the Universe. London: Jonathan Cape, 2004.

[32] Maldacena J, Susskind L. Cool horizons for entangled black holes. Fortschritte der Physik 2013; 61(9): 781-811. arXiv:1306.0533, doi:10. $1002 /$ prop. 201300020

[33] Bhaumik ML. Reality of the wave function and quantum entanglement. 2014. arXiv: 1402.4764

[34] van Raamsdonk M. Building up spacetime with quantum entanglement. General Relativity and Gravitation 2010; 42(10): 2323-2329. arXiv: 1005.3035, doi : $10.1007 / \mathrm{s} 10714-010-1034-0$

[35] Swingle B. Constructing holographic spacetimes using entanglement renormalization. 2012. arXiv: 1209.3304

[36] Einstein A, Schrödinger E, Planck M, Lorentz HA. Letters on Wave Mechanics. Przibram K (editor), New York: Philosophical Library, 1967.

[37] Aspect A, Grangier P, Roger G. Experimental realization of Einstein-Podolsky-Rosen-Bohm Gedankenexperiment: A new violation of Bell's inequalities. Physical Review Letters 1982; 49(2): 91-94. doi : 10.1103/PhysRevLett.49.91

[38] Aspect A, Dalibard J, Roger G. Experimental test of Bell's inequalities using time-varying analyzers. Physical Review Letters 1982; 49(25): 1804-1807. doi : 10.1103/PhysRevLett.49.1804

[39] Bennett CH, Brassard G. Quantum cryptography: public key distribution and coin tossing. Proceedings of the IEEE International Conference on Computers, Systems and Signal Processing, Bangalore,
India, December 10-12, 1984, pp. 175-179. doi: $10.1016 /$ j.tcs. 2014.05 .025

[40] Zurek WH. Decoherence and the transition from quantum to classical. Physics Today 1991; 44(10): 36-44. doi: 10.1063/1.881293

[41] Zurek WH. Decoherence and the transition from quantum to classical-revisited. Los Alamos Science 2002; 27: 86-109. arXiv:quant-ph/0306072, http://library, lanl.gov/cgi-bin/getfile?27-09.pdf

[42] Zurek WH. Decoherence, einselection, and the quantum origins of the classical. Reviews of Modern Physics 2003; 75(3): 715-775. arXiv:quant-ph/ 0105127, doi : 10.1103/RevModPhys.75.715

[43] Zurek WH. Probabilities from entanglement, Born's rule $p_{k}=\left|\psi_{k}\right|^{2}$ from envariance. Physical Review A 2005; 71(5): 052105. arXiv:quant-ph/0405161, doi:10.1103/PhysRevA.71.052105

[44] Schlosshauer M. Decoherence and the QuantumTo-Classical Transition. The Frontiers Collection, Elitzur AC, Silverman MP, Tuszynski JA, Vaas R, Zeh H-D (editors), Berlin: Springer, 2007. doi: 10. $1007 / 978-3-540-35775-9$

[45] Schlosshauer M. Decoherence, the measurement problem, and interpretations of quantum mechanics. Reviews of Modern Physics 2005; 76(4): 1267-1305. arXiv:quant-ph/0312059, doi:10. 1103/RevModPhys.76.1267

[46] Schlosshauer M, Fine A. On Zurek's derivation of the Born rule. Foundations of Physics 2005; 35(2): 197-213. arXiv:quant-ph/0312058, doi:10. 1007/s10701-004-1941-6

[47] Haroche S. Controlling photons in a box and exploring the quantum to classical boundary. Nobel Lecture, December 8, 2012. https://www nobelprize.org/nobel_prizes/physics/ laureates/2012/haroche-lecture.pdf

[48] Wineland DJ. Superposition, entanglement, and raising Schrödinger's cat. Nobel Lecture, December 8, 2012. https://www.nobelprize.org/ nobel_prizes/physics/laureates/2012/ wineland-lecture.pdf

[49] Monroe C, Meekhof DM, King BE, Wineland DJ. A "Schrödinger cat" superposition state of an atom. Science 1996; 272(5265): 1131-1136. doi : 10.1126/ science.272.5265.1131 
[50] Zhang J, Pagano G, Hess PW, Kyprianidis A, Becker P, Kaplan H, Gorshkov AV, Gong ZX, Monroe C. Observation of a many-body dynamical phase transition with a 53-qubit quantum simulator. $\mathrm{Na}$ ture 2017; 551(7682): 601-604. doi:10.1038/ nature24654

[51] Song C, Xu K, Liu W, Yang C-P, Zheng S-B, Deng H, Xie Q, Huang K, Guo Q, Zhang L, Zhang P, Xu D, Zheng D, Zhu X, Wang H, Chen YA, Lu CY, Han S, Pan J-W. 10-qubit entanglement and parallel logic operations with a superconducting circuit. Physical Review Letters 2017; 119(18): 180511. arXiv: 1703.10302, doi:10.1103/PhysRevLett.119. 180511

[52] Shenoy-Hejamadi A, Pathak A, Radhakrishna S. Quantum cryptography: key distribution and beyond. Quanta 2017; 6(1): 1-47. doi:10.12743/ quanta.v6i1.57

[53] Engel GS, Calhoun TR, Read EL, Ahn T-K, Mancal T, Cheng Y-C, Blankenship RE, Fleming GR. Evidence for wavelike energy transfer through quantum coherence in photosynthetic systems. $\mathrm{Na}$ ture 2007; 446(7137): 782-786. doi:10.1038/ nature05678

[54] Lee H, Cheng Y-C, Fleming GR. Coherence dynamics in photosynthesis: protein protection of excitonic coherence. Science 2007; 316(5830): 1462-1465. doi : $10.1126 /$ science .1142188

[55] Calhoun TR, Ginsberg NS, Schlau-Cohen GS, Cheng Y-C, Ballottari M, Bassi R, Fleming GR. Quantum coherence enabled determination of the energy landscape in light-harvesting complex II. Journal of Physical Chemistry B 2009; 113(51): 16291-16295. doi:10.1021/jp908300C

[56] Collini E, Wong CY, Wilk KE, Curmi PMG, Brumer $\mathrm{P}$, Scholes GD. Coherently wired light-harvesting in photosynthetic marine algae at ambient temperature. Nature 2010; 463(7281): 644-647. doi : 10.1038/ nature08811

[57] Panitchayangkoon G, Hayes D, Fransted KA, Caram JR, Harel E, Wen J, Blankenship RE, Engel GS. Long-lived quantum coherence in photosynthetic complexes at physiological temperature. Proceedings of the National Academy of
Sciences 2010; 107(29): 12766-12770. doi:10. 1073 /pnas. 1005484107

[58] Sarovar M, Ishizaki A, Fleming GR, Whaley KB. Quantum entanglement in photosynthetic light-harvesting complexes. Nature Physics 2010; 6(6): 462-467. arXiv:0905.3787, doi: $10.1038 /$ nphys 1652

[59] Ishizaki A, Fleming GR. Quantum superpositions in photosynthetic light harvesting: delocalization and entanglement. New Journal of Physics 2010; 12(5): 055004. doi: 10. 1088/1367-2630/12/5/ 055004

[60] Whaley KB, Sarovar M, Ishizaki A. Quantum entanglement phenomena in photosynthetic light harvesting complexes. Procedia Chemistry 2011; 3(1): 152164. doi : 10.1016/j.proche.2011.08.021

[61] Gauger EM, Rieper E, Morton JJL, Benjamin SC, Vedral V. Sustained quantum coherence and entanglement in the avian compass. Physical Review Letters 2011; 106(4): 040503. arXiv:0906.3725, doi:10.1103/PhysRevLett.106.040503

[62] Hameroff SR, Penrose R. Consciousness in the universe: a review of the 'Orch OR' theory. Physics of Life Reviews 2014; 11(1): 39-78. doi:10.1016/j. plrev.2013.08.002

[63] Arndt M, Nairz O, Vos-Andreae J, Keller C, van der Zouw G, Zeilinger A. Wave-particle duality of $\mathrm{C}_{60}$ molecules. Nature 1999; 401(6754): 680-682. doi: $10.1038 / 44348$

[64] Eibenberger S, Gerlich S, Arndt M, Mayor M, Tüxen J. Matter-wave interference of particles selected from a molecular library with masses exceeding 10000 amu. Physical Chemistry Chemical Physics 2013; 15(35): 14696-14700. doi: $10.1039 / \mathrm{c} 3 \mathrm{cp} 51500 \mathrm{a}$

[65] O'Connell AD, Hofheinz M, Ansmann M, Bialczak RC, Lenander M, Lucero E, Neeley M, Sank D, Wang H, Weides M, Wenner J, Martinis JM, Cleland AN. Quantum ground state and singlephonon control of a mechanical resonator. $\mathrm{Na}$ ture 2010; 464(7289): 697-703. doi:10.1038/ nature08967 